\title{
PQLI Definition of Criticality
}

\author{
Roger Nosal • Tom Schultz
}

Published online: 19 May 2008

(C) International Society for Pharmaceutical Engineering 2008

\begin{abstract}
This paper describes progress made by the Criticality Task Team within the ISPE PQLI initiative. It aims to provide a concise, coherent, and universal approach for determining criticality for parameters, material attributes, conditions, and quality attributes. The work also clarifies the risk based distinctions governing the assignment of criticality to provide consistency and facilitate the adoption and implementation of Quality by Design (QbD) principles in the development of pharmaceutical manufacturing processes. The application of the concept of criticality presented in this paper aligns with the principles of ICH Q8, Q9 and Q10 guidelines.
\end{abstract}

Keywords PQLI $\cdot$ Design space $\cdot$ Criticality $\cdot$ Control strategy $\cdot$ Critical quality attribute $\cdot$ Critical process parameter Knowledge space $\cdot$ Target product profile . Product and process knowledge · Quality by design . Quality risk management

Contributing Team Members: Joanne Barrick - Lilly, Chris Brook Glaxo Smith Kline, Dwayne Campbell - GlaxoSmithKline, Ranjit Deshmukh - Wyeth, John Donaubauer - Abbott, John Groskoph Pfizer, Zena Kauffman - Abbott, Mette Kraemer Hansen -

NovoNordisk, Jay Lakshman - Novartis, Wendy Mavroudakis Johnson \& Johnson, Garry O'Connor - Pfizer, Wim Oostra - Organon, Shering-Plough, Steve Simmons - Wyeth, Shailesh Singh- Wyeth, Manny Sundararajan - Astra Zeneca, Kevin Wall - Noramco, Tim Watson - Pfizer

\section{R. Nosal}

Pfizer Inc.,

Eastern Point Rd.,

Groton, CT 06340, USA

e-mail: roger.nosal@pfizer.com

\section{T. Schultz}

Johnson \& Johnson,

1125 Trenton-Harbourton Rd.,

Titusville, NJ 08560, USA

e-mail: tschultz@prdus.jnj.com

\section{Introduction}

The word "critical" is an adjective and its use to describe pharmaceutical quality or manufacturing process variables requires context. ${ }^{1}$ The use of the word "critical" to define a process variable, step or attribute can be subjective, relying on individual assessment of relative risk, based on empirical knowledge, direct experience or data. Therefore, its meaning is neither universal nor definitive. In fact, industry concerns regarding the regulatory implications of labeling a process variable, attribute or step as "critical" have produced a variety of cautiously crafted and detailed definitions for its use. This position paper was developed and written to provide a concise, coherent and universal approach for delineating criticality for process variables, e.g., parameters, material attributes, conditions, etc., and quality attributes. The purpose of this position paper is to clarify the risk based distinctions governing the assignment of criticality to provide some measure of consistency and facilitate the adoption and implementation of Quality by Design $(\mathrm{QbD})$ principles in the development of pharmaceutical manufacturing processes.

The assessment of criticality as it relates to pharmaceutical quality is generally determined as a function of risk. However a legitimate risk assessment is neither effective nor confident when made in the absence of product knowledge or process understanding and experience. The pharmaceutical industry has traditionally assessed risk, particularly during development, based predominantly on empirical knowledge, understanding derived from manufacturing experience. The advent of Quality by Design has shifted the paradigm from a retrospective to a prospective,

\footnotetext{
${ }^{1}$ Crit·i-cal $a d j$ : of essential importance. Several definitions for the word "critical" exist, each reflective of the word it modifies. The application of criticality presented in this abstract aligns with principles articulated in ICH Q8, Q9 and Q10 guidelines.
} 
systematic and risk-based approach to develop process understanding. The need to prioritize and focus experiments on meaningful variable interactions, to characterize relevant design space boundaries and establish appropriate control strategies warrants a robust definition of criticality.

While a simple unencumbered definition of "critical" might improve consistency in its application, it would also limit the continuum of risks ascribed to criticality that encompasses a myriad of relatively complicated, yet important, business and quality designations for process variables and product attributes. Figure 1 provides an example of one company's internal approach to define critical process parameters. The variety of designations reflects how the pharmaceutical industry characterizes criticality to coincide with multiple expectations from quality, regulatory and business stakeholders. In addition, the degree of criticality as a direct reflection of risk may be conservative depending on the objective of the assessment.

The ISPE PQLI Criticality Task Team continues to gather collective experience from industry and regulatory authorities to understand the significance of criticality and its application to evaluate variables, process parameters as well as product and material attributes. ${ }^{2}$ In general, both industry and regulatory authorities acknowledge distinctions in the definition of critical variables particularly where there was regulatory impact. Nevertheless, several common and consistent technical assumptions have emerged from this feedback:

- Criticality can describe any feature or material attribute, property or characteristic of a drug substance, component, excipient, drug product or device and/or any process attribute, parameter, condition or factor in the manufacture of a drug product.

- Criticality Analysis distinguishes non-critical variables from both known and potentially critical variables while delineating levels or degrees of risk (e.g. high or low) by which known and potentially critical sources of variability which may impact the quality of the product are assessed.

- Critical impact may be attributed to multiple variables operating in sequence or in concert.

- As a result of a Criticality Analysis, designation of noncritical and critical categories for process variables and/or product attributes provides a relative and qualitative classification of their impact to quality, and by extension to safety and efficacy ${ }^{3}$ of the drug product for the patient.

- A control can reduce the level of criticality but it does not change the potential for severity of harm/impact

\footnotetext{
${ }^{2}$ The substrate for the preliminary position described herein was developed through direct engagement of industry representatives and solicited comments from representatives from FDA, EMEA and MHLW at various ISPE conferences [1-3].

${ }^{3}$ Reference is made to ICH Q8R [4] and ICH Q6A [5] wherein demonstration of quality is commensurate with reducing the impact to safety and efficacy of the product for the patient.
}

even though it may reduce the probability and/or increase detectability.

- A control that reduces the level of criticality for a process variable, by definition, becomes a critical control point /parameter/ (HACCP).

- A few drug product CQAs, such as sterility, will always be critical. These CQAs may be dosage form specific.

- Criticality Analysis is relevant in development of design space and design space boundaries and establishing effective control strategies.

- The Criticality Analysis Decision Tree differentiates business decisions, categorizes non-critical variables in accordance with risk and, at the discretion of the sponsor, provides flexibility to designate levels of criticality governed by relative levels of risk.

- As attribute or parameter boundaries approach edges of failure the level of criticality increases with an increase in the level of risk.

- Process parameters or variables controlled to evaluate business attributes are not necessarily critical unless demonstrated to directly or indirectly impact quality of the drug product.

Delineating criticality is essentially a function of measured risk assessment as shown in Fig. 2. The assessment of risk is fundamentally predicated on understanding the relationship of process variables to one another and their collective impact to quality attributes of the product. A variable that is non-critical is differentiated, or categorized, from variables that are potentially critical relative to the severity and probability of risk that variable poses to safety, efficacy and quality of the product and harm to the patient. The relative level or degree of risk these variables pose is assessed relative to the probability of occurrence and ability to detect failure and impose potential harm to the product/patient.

The assessment of criticality is not static. Continuous process improvement is a presumptive expectation of Quality by Design. Innovations, adjustments, optimization and modifications warrant reassessment of risk and criticality. Therefore risk management is central to the delineation of criticality and includes the following elements as summarized from ICH Q9 [6]:

- Risk Identification - finding what risks exist and/or might exist — problems and potential problems

- Risk Evaluation - severity, probability and detectability

- Risk Control - detectability, i.e., risk management versus risk measurement and monitoring

$>$ Risk Reduction-well grounded science and justifiable decision making to the extent necessary to provide appropriate assurance and high degree of probability of safety, efficacy and quality 


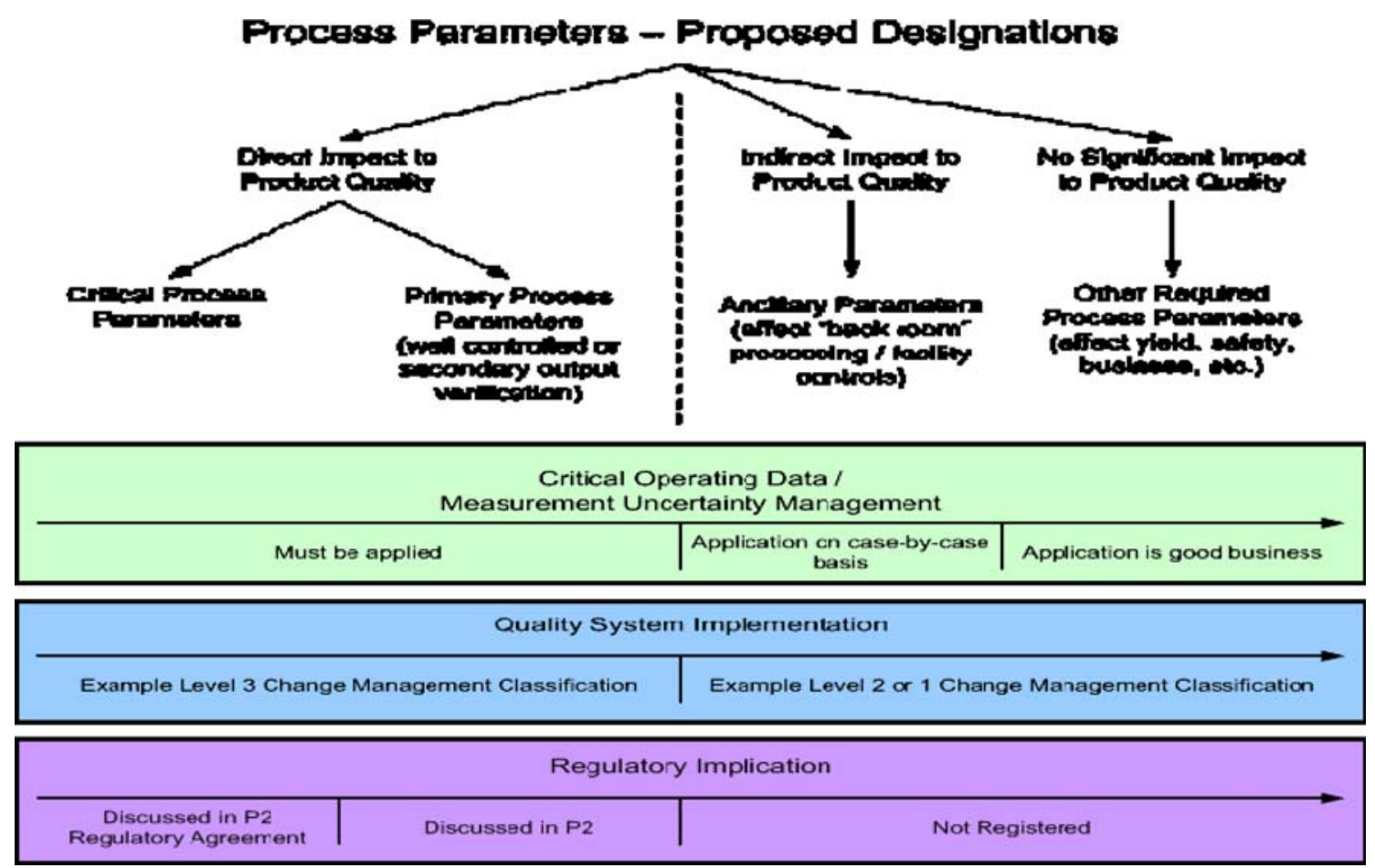

Fig. 1 Example of designations for criticality

$\triangle$ Risk Mitigation-development of design space, controls and/or measurements to demonstrate reduction in the severity of risk.

$>$ Risk Acceptance-

$\checkmark$ Some level of risk may remain

$\checkmark$ Some level of risk may be appropriate considering the benefit accrued

$\checkmark$ Some level of risk may be practicably achievable

$\checkmark$ Acceptance of residual risk in accordance with benefit for patient

- Risk Communication -

$\triangleright$ 'Customers' need to know what risks have been considered, how they have been managed and/or controlled and what residual risk has been accepted.

$\triangleright$ Risk communication can also be a strategic part of a risk management program

Based on these functional precepts, the development of a Criticality Analysis Decision Tree emerged as a relatively direct, non-prescriptive risk assessment process by which variables, attributes and parameters may be categorized. Variables with potential to have direct impact on safety and efficacy as well as quality are distinguished from those variables, attributes and parameters that do not have an impact on safety and efficacy and are generally associated with business criteria, i.e., variables associated with process efficiency. This process describes a relatively high level Criticality Analysis that initially distinguishes potentially critical from non-critical variables with subsequent provisions to differentiate levels of criticality based on formal risk assessment through the use of "Risk Filters". The Criticality Analysis Decision Tree is presented in Fig. 3.

The criticality decision tree is divided into two decision classifications to facilitate Criticality Analysis. The top classification is labeled Foundational and serves as the basis for subsequent decisions delineating critical from non-critical variables. In the absence of a modern risk-based quality system, a traditional specification may serve as the basis for determining critical and non-critical variables and is typically demonstrated by empirical, process experience. The decision to develop a control strategy based on traditional specification limits without benefit of multivariate analysis is driven by business needs at the discretion of the sponsor.

Similarly, a decision to proceed with a risk-based, systematic and prospective approach to pharmaceutical

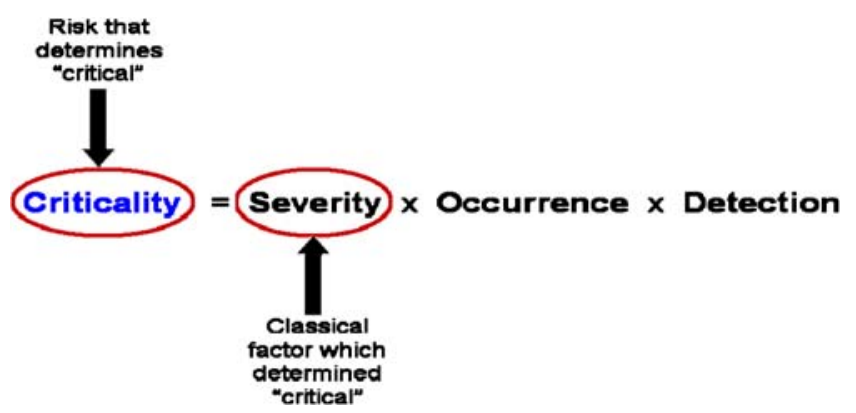

Fig. 2 Fundamental elements of risk assessment 


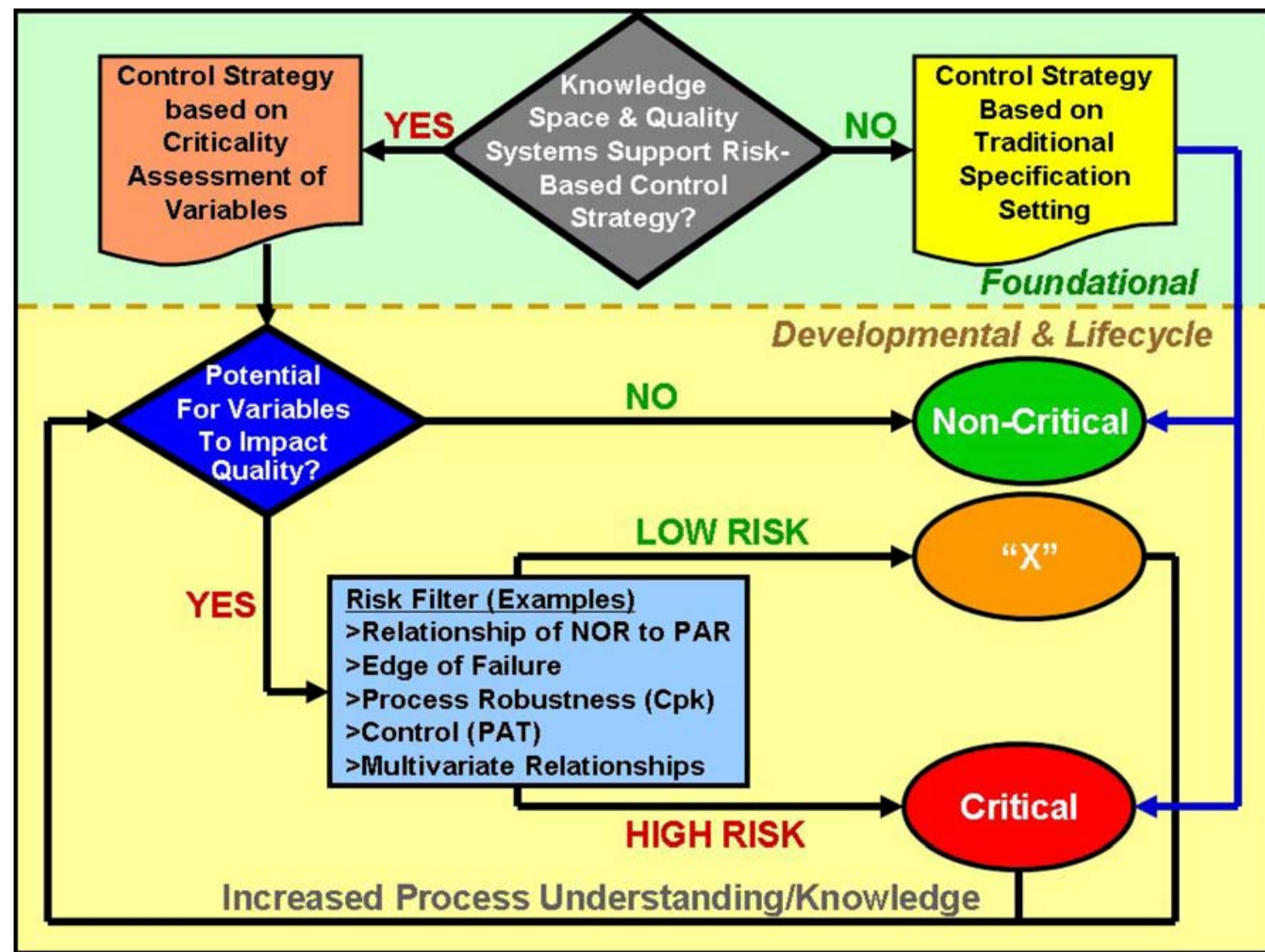

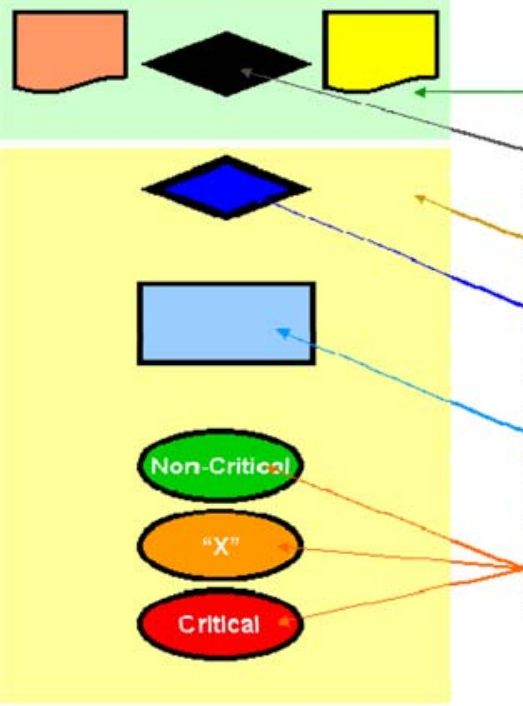

Fig. 3 Criticality analysis decision tree

development relies on evaluating risk relative to impact on safety, efficacy and quality. This categorization differentiates non-critical from potentially critical variables, parameters and attributes based primarily on the severity or magnitude of the risk. Subsequently, a variable, parameter or attribute is assessed to delineate levels of criticality based on whether the probability and the ability dotoctability
NOR = Normal Operating Range PAR = Proven Acceptable Range PAT = Procees Analytleal Technology

Foundational Classification - Bus/ness decision

Business Declslon - determInes QbD vs. traditional Developmental \& Lifecycle Classiflcation - Qualhy declsion

Risk Assessment Declsion - categorizes non-critical from otentially critical based on severity \& probability of relatlve risk (high or low), l.e., probablitty \&

Criticality Designations - determined after appropriate isk assessment

to detect the risk is low or high. Provisions in this process suggest the use of a Risk Filter to discriminate levels of risk once a variable is categorized as potentially critical. The Risk Filter identifies several examples (by no means exhaustive) to provide the basis to evaluate relative risk.

The level of criticality labeled " $X$ " allows for classification of variables, parameters and attributes for which risk 
(probability and detectability) has not been definitively ascertained or is secondary or ancillary to other factors. Variables, parameters and attributes labeled " $\mathrm{X}$ " may require re-evaluation when making changes to multi-variate design space. In addition, more than two levels of criticality may be used to describe the level of risk for variables labeled "X".

The Criticality Analysis Decision Tree is applicable to API, drug product, biological and device processes and products. The integrity of the risk assessment is predicated on a robust quality system in alignment with principles articulated in ICH Q9 and Q10 [7] and dependent on well developed knowledge space for the manufacturing process. As process understanding and knowledge increase during the lifecycle of a product the delineation of criticality serves as an iterative process to reassess the risk of process variables and quality attributes.

\section{Detailed Explanation of Criticality Analysis Decision} Tree

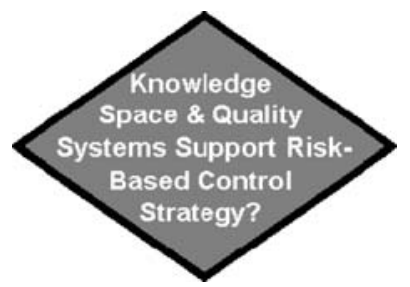

The first decision differentiates "traditional" from "QbD" approaches. The difference in approaches is in the depth and quality of process understanding achieved and demonstrated. QbD approaches tend to establish multi-variate design space boundaries and holistic control strategies while a traditional approach relies predominantly on process experience, rigid processing and reliance on end product testing for control. The differentiation between the two approaches is articulated in $\mathrm{ICH} \mathrm{Q(8)}$ R. $[4]^{4}$ The decision to proceed with a risk-based control strategy to develop product and process understanding will align with the QbD approach described in ICH Q(8)R. The decision to proceed with a "traditional" approach sug- gests adherence to control criteria described in ICH Q6A [5].

\footnotetext{
4 "A more systematic approach to development (also defined as quality by design) can include, for example, incorporation of prior knowledge, results of studies using design of experiments, use of quality risk management, and use of knowledge management (see ICH Q10) throughout the lifecycle of the product. Such a systematic approach can enhance the process to achieve quality and help the regulators to better understand a company's strategy. Product and process understanding can be updated with the knowledge gained over the product lifecycle."
}

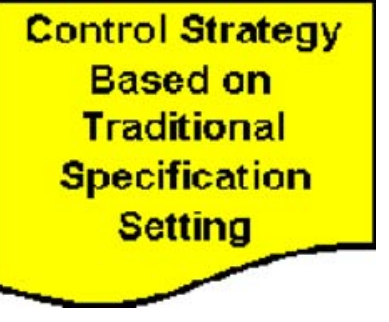

The "traditional" approach differentiates critical from non-critical attributes and parameters relative to traditional CQA's defined by specifications. Parameters designated as "critical" warrant demonstration of control confirmed by an appropriate test method based on process experience.

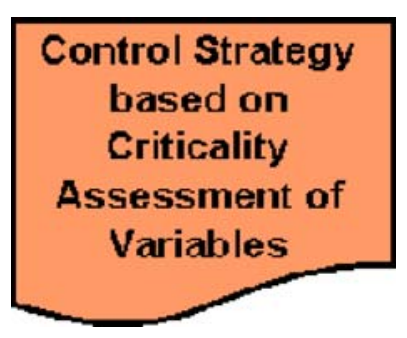

Where a risk-based control strategy is developed, categorization of variables as noncritical or critical can be delineated by appropriate risk assessment and experimentation, i.e., the "QbD approach." The "QbD" approach leads to the following collective expectations:

- Product quality and performance are ensured through the design of effective and capable, i.e., robust manufacturing processes

- Product and process specifications are based on a mechanistic understanding of how formulation and process factors affect product quality and performance. ${ }^{5}$

- Continuous product and process improvement

- Relevant regulatory policies and procedures are tailored to reflect current level of scientific knowledge and associated risk

- Risk-based regulatory approaches recognize:

$\triangleright$ the level of scientific understanding of how formulation and manufacturing process factors affect product quality and performance

$>$ the capability of process control strategies to prevent or mitigate the risk of producing a poor quality product

Prior knowledge is an appropriate basis for risk assessment decisions for new and legacy products that proceed through the "QbD" approach.

The bottom layer of the criticality decision tree process introduces the risk assessment strategy employed by a QbD approach to product and process development. This decision tree can be used to assess the criticality of variables in a product or process, i.e., attributes and parameters. The blue decision diamond requesting a response to the question, "What is the potential for variables to impact quality?" distinguishes the first "category of criticality" by segregating variables that do not have an impact on quality and designating them non-critical.

\footnotetext{
${ }^{5}$ A specification is not synonymous to control strategy. A specification is only one element of a control strategy.
} 
Those variables that may have an impact on quality fall into the second "category of criticality" which can be subsequently evaluated through appropriate risk assessment, as denoted by the "Risk Filter" into "levels of criticality" designated as either critical or some intermediate level " $\mathbf{X}$ " defined by the magnitude and attenuation of severity, probability and detectability of risk.

Attributes ${ }^{6}$ fall within two major categories, those that are the measurable properties of a material and those that are the measurable characteristics of the process used to manufacture a material. Quality Attributes have an impact on patient safety, efficacy or quality. An attribute should be defined as a Quality Attribute where precedented pharmaceutical knowledge suggests a link may exist even if a direct, scientifically demonstrable link has not been made between the attribute and patient safety, efficacy and/or quality.

Variables or parameters that may have important implications for business purposes, i.e., reducing costs, optimizing yield, improving process efficiency, environmental and/or worker safety, etc., or variables associated with a delivery device and/or packaging of the drug product. may be assessed for their level of criticality relative to business criteria. However these variables are not, by definition, necessarily critical with regards to safety and/or efficacy and should not be defined as critical from a regulatory perspective.

Process parameters ${ }^{7}$ are variables that have either been demonstrated to have a significant impact on Quality Attributes of the API and/or drug product or those that have been demonstrated not to have a significant impact on the Quality Attributes of the API and/or drug product.

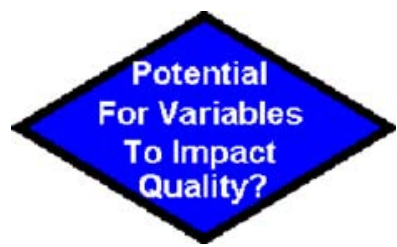

Elements necessary to perform a Criticality Analysis, (i.e., risk assessment tools, functional relationships to critical quality attributes, and the criteria by which a variable is designated as critical, in combination with other variables or independently), may be determined from (1) prior knowledge, (2) results from concerted and prospective designed (e.g., multi-variate) experimentation and (3) demonstrated process experience. The quality of a product as defined in accordance with ICH Q $6 \mathrm{~A}^{8}$ is a minimum standard and is a reflection of the Target Product Profile. The Target Product Profile describes the quality attributes

\footnotetext{
${ }^{6}$ At·tri·bute $n$ : A quality or characteristic inherent in or ascribed to someone or something.

${ }^{7}$ Pa.ram-e.ter $n$. One of a set of measurable factors, such as temperature and pressure, that define a system and determine its behavior and are varied in an experiment.

8 "The definition of quality is the suitability of either a drug substance or drug product for its intended use."
}

of the product for its intended use and administration. While an assessment of risk is predicated on the established foundation of a robust quality system as defined in accordance with ICH Q (10), the primary assessment and designation of criticality should be made relative to impact to safety and efficacy of the drug product to the patient which are dependent on measures of quality as reflected by its Quality Attributes. Development and establishment of product Quality Attributes to meet the Target Product Profile introduces a standard level of quality, the measured assessment of which will establish critical quality attribute (CQA) boundaries A well justified risk assessment identifies criteria to establish the appropriate and relevant elements of a control strategy. The quality of a product is measured by the demonstrated application of that control strategy.

Process parameters are assessed for criticality relative to their impact on product Quality Attributes. Typically, a vast parameter-attribute matrix is identified and assessed in accordance with prior knowledge to establish a level of risk for each parameter relative to its respective Quality Attributes. Experiments may be conducted in parts of that variable space where the functional relationship between parameters and attributes is not well understood. These experiments establish functional relationships to describe that variable space and demonstrate process understanding. Results from this exercise provide criteria by which an appropriate risk assessment can be justified. There are at least two categories of criticality to establish risk, i.e., critical and non-critical. When it is demonstrated or suspected that a variable may influence quality, the level of risk (High or low) is determined by the application of tools in the Risk Filter. The Risk Filter is not exhaustive and many other tools may be used to evaluate impact of risk and delineate criticality. A process is considered well understood from the physico-chemical perspective when

- $\quad$ sources of variability are explained

- "Quality Attributes" can be predicted based on key inputs, e.g., process parameters, material attributes, etc. - process capability of "Quality Attributes" meets acceptance levels

If a variable does not have potential to impact Quality, it is Non-Critical. If a variable has the potential to impact Quality, the magnitude of the potential for risk must be evaluated by application of appropriate measures of risk.

Risk Filter (Examples)
>Relationship of NOR to PAR
>Edge of Failure
>Process Robustness (Cpk)
>Control (PAT)
>Multivariate Relationships

The application of principles of Quality by Design in the development of process understanding is predicated on risk assessment. Risk assessments provide an evaluation of process variables relative to their level of expected impact on quality. 
Risk assessment is fundamental to the delineation of criticality in the evaluation of process dimensions and development of design space. The principles and several tools for conducting risk assessments are described in ICH $\mathrm{Q}$ (9). ${ }^{9}$ Criticality is a function of the probability, severity and detectablity of a variable's impact on the quality, in particular, quality attributes, of a product and the detectability for changes in that variable. In FMEA, the product of Probability X Severity X Detectability is referred to as the Risk Priority Number. In general, risk should be assessed based on cause and effect and relative to the following criteria:

- Probability - the likelihood of an occurrence.

- Severity - the magnitude of the impact of a consequence.

- Detectability - the level or ability at which an occurrence can be measured.

- Sensitivity (optional) — the attenuation of interactions between multivariate dimensions.

In addition, due consideration should be given to the following factors that may be used to assess risk:

- There are differences in designation of criticality and level of risk between API and drug product parameters.

- Critical outcomes may be attributed to multiple variables operating in concert.

- Control strategy based solely on testing does not make a CQA or CPP non-critical; it just makes it controlled. The severity of the risk does not change with an assurance of control, only the probability of risk is reduced with an assurance of control.

- Use of descriptive adjectives to define criticality should include context.

- Several levels of criticality may be used to describe multiple levels of risk.

- As attribute or parameter boundaries approach edges of failure the level of criticality increases with the level of risk.

- Criticality is intended to be revisited and assessed during product lifecycle.

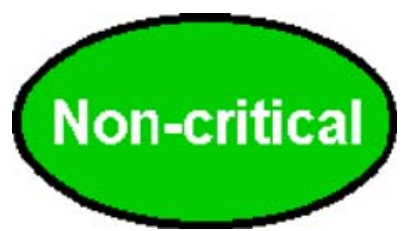

Attributes, parameters and other variables that have either been demonstrated or based on prior knowledge known not to have potential to impact safety and/or efficacy of the product are categorized as Non-Critical. NonCritical attributes, parameters and variables do not pose a

\footnotetext{
${ }^{9}$ ICH Q (9) Quality Risk Management
}

risk because they are not functionally related to Critical Quality Attributes. In accordance with ICH Q (8) R noncritical quality attributes and process parameters do not have to be included in descriptions of design space. While the classification of non-critical variables should be justified, and where appropriate substantiated by data, additional scientific or technical evaluations of these variables are unwarranted.

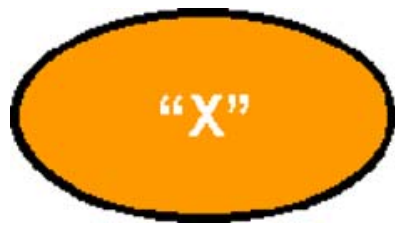

" $\mathrm{X}$ " represents an alternative designation for attributes, parameters or variables that have either been demonstrated, or based on prior knowledge, suspected to have the potential to impact the quality of the product, where the level of risk has been assessed as low. This designation may also be employed to delineate attributes, parameters or variables:

- that may have an indirect impact on safety and/or efficacy by themselves or in combination with other variables,

- where risk is mitigated

- where demonstration of knowledge transfer for noncritical variables requires additional evaluation.

Attributes, parameters or variables in this category may be functionally related to CQA's or other quality attributes. The potential impact or risk these parameters or attributes pose is re-evaluated in accordance with relevant changes to the manufacturing process.

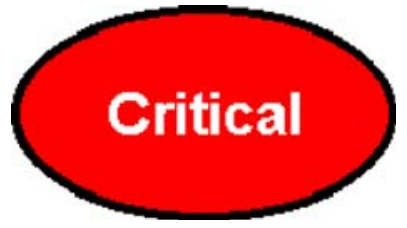

A material or process attribute or parameter that is categorized as critical has either been demonstrated or based on prior knowledge known to have a direct or indirect impact on patient safety, therapeutic efficacy, in vivo pharmacokinetic/pharmacodynamic performance and patient compliance for which failure to control within demonstrable acceptance criteria produces an unrecoverable result. A Critical Process Parameter CPP is a process input that, when varied beyond a limited range, has a direct and significant influence on a CQA. A Critical Quality Attribute is a quantifiable property of an intermediate or final product that is considered critical for establishing the intended purity, efficacy, and safety of the product. That is, the property must be within a predetermined range to ensure final product quality. Risk is controlled for a CQA by establishing a product specification for the affected attribute. See ICH Q8 Pharmaceutical Development [8] 
Lifecycle Management — Increased Product/Process Understanding

During the lifecycle of a product there is a presumption that an increase in process understanding and product knowledge derived from continuous improvement and optimization activities as well as increased manufacturing experience may require re-assessment of attributes, parameters and other variables. In accordance with ICH Q9 definition of risk management the Criticality Analysis Decision Tree should be used for periodic reassessment. Variables, parameters and attributes implicated in changes to manufacturing processes or product criteria should be evaluated relative to relevant quality attributes. Changes to material or process attributes or parameters controlled to evaluate business criteria are not necessarily treated as critical unless there is a demonstrated link to safety, efficacy and quality of the drug product. Changes in designation from one level or category of criticality to another must be demonstrated by data or increased knowledge, i.e., modeling and improved interpretation of already existing data etc., and may require appropriate notification to regulatory authorities.

Throughout the lifecycle of a product, control strategy should differentiate levels of criticality depending on the impact to the quality of the drug product as defined by the relationship of process variables and parameters and material attributes to quality attributes.

\section{Glossary}

1. Attribute - A characteristic or inherent property of feature. (ASTM E2363-06a)

2. Change Management - A systematic approach to proposing, evaluating, approving, implementing and reviewing changes. (ICH Q10 EWG)

3. Continuous Verification - An approach to process validation in which manufacturing process performance is continuously monitored and evaluated. (ICH Q9)

4. Control Strategy - A planned (like word 'planned') set of controls, derived from current product and process understanding, that assures process performance and product quality. The controls can include parameters and attributes related to drug substance and drug product materials and components, facility and equipment operating conditions, in-process controls, finished product specifications, and the associated methods and frequency of monitoring and control. (ICH Q10 EWG)

5. Critical (For Small Molecules Only) - Critical describes a feature or aspect of a raw material, intermediate, process or product that is generally identified through an assessment of the extent to which its variation can have impact on the quality of the drug product, and which therefore requires control to ensure that the product is of acceptable quality. (ICH Q8R) v5a. Critical (For Biologics Only) - An input parameter that must be controlled within a meaningful, narrow range to ensure that drug substance product Critical Quality Attributes meet specifications.

6. Critical Process Parameter - A process input that, when varied beyond a limited range, has a direct and significant influence on a CQA. (PQRI Robustness Paper)

7. Critical Quality Attribute - A quantifiable property of an intermediate or final product that is considered critical for establishing the intended purity, efficacy, and safety of the product. (ICH Q8R)

8. Design Space - The multidimensional combinations and interaction of input variables (e.g. material attributes) and process parameters that have been demonstrated to provide assurance of quality. Working within the design space is not considered as a change. Movement out of the design space is considered to be a change and would normally initiate a regulatory post-approval change process. Design space is proposed by the applicant and is subject to regulatory assessment and approval. (ICH Q8)

9. Design of Experiments (DoE) - The arrangement in which an experimental program is to be conducted, and the selection of the levels (versions) of one or more factors or factor combinations to be included in the experiment.

9a. Formal Experimental Design - A structured, organized method for determining the relationship between factors affecting a process and the output of that process. Also known as "Design of Experiments". (ICH Q8)

10. Detectability - The ability to discover or determine the existence, presence, or fact of a hazard. (ICH Q9)

11. Edge of Failure - the boundary to a variable or parameter, beyond which the relevant quality attributes or specification cannot be met. (ICH Q8R)

12. Innovation - The introduction of new technologies or methodologies to pharmaceutical development and manufacturing. (ICH Q10 EWG)

13. Harm - Damage to health, including the damage that can occur from loss of product quality or availability.

14. " $\mathrm{X}$ " Key - There is managed risk relative to impacting the safety or efficacy of a product; well controlled.

15. Key Process Parameter (KPP) (For Small Molecules Only) - Process parameters that may impact a critical quality attribute. In contrast to a critical process parameter, a key process parameter has a reduced risk of impacting the safety or efficacy of the product, even though there is a relationship to a critical quality attribute. The risk of failure is mitigated by applying a robust proactive control strategy through internal quality management system to reduce the natural variability of the parameter in question. Operating ranges for key process parameters may be filed as regulatory commitments. 
15a. Key Process Parameter (KPP) (For Biologics Only) - An adjustable parameter (variable) of the process that, when maintained within a narrow range, ensures optimum process performance. A key process parameter does not affect critical product quality attributes.

16. Knowledge Management - Systematic approach to collecting, analyzing, storing, and disseminating information related to products, processes and components. (ICH Q10 EWG)

17. Lifecycle - All phases in the life of a product from the initial development through marketing until the product's discontinuation. Synonymous with Product Lifecycle. (ICH Q8)

18. Non-Critical - There is a negligible risk of impacting the safety or efficacy of a product. Syn: Regulatory Insignificant. 19. Non-Critical Process Parameter (For Small Molecules Only) - A process parameter that has a negligible risk of impacting the safety or efficacy of a product. It impacts NonCritical Quality Attributes which are not related to the safety or efficacy of a product. Non-Critical Process Parameters are managed only by internal quality and change control systems. 19a. Non-Critical Process Parameter (For Biologics Only) All input parameters that fall outside of the definition for critical process parameters are non-critical. Non-critical process parameters may be further subdivided into Key and Non-Key process parameters, in cases where process performance impact is demonstrated.

20. Non-Critical Quality Attribute - A quality attribute that has a low risk of impacting the safety or efficacy of a product. Non-Critical Quality Attributes are managed only by internal quality and change control systems.

21. Non-Key Process Parameter (non-KPP) (For Biologics Only) - An input parameter that has been demonstrated to be easily controlled or has a wide acceptable limit.

22. Normal Operating Range (NOR) - A defined range, within the Proven Acceptable Range, specified in the manufacturing instructions as the target and range at which a process parameter is controlled, while producing unit operation material or final product meeting release criteria and Critical Quality Attributes. (PQRI Robustness Paper)

23. Parameter - A measurable or quantifiable characteristic of a system or process. (ASTM E2363-06a)

24. Pharmaceutical Quality System - Management system to direct and control a pharmaceutical company with regard to quality. (ICH Q10 EWG based upon ISO 9000-2005)

25. Process Analytical Technologies (PAT) - A system for designing, analyzing, and controlling manufacturing through timely measurements (i.e., during processing) of critical quality and performance attributes of raw and in-process materials and processes with the goal of assuring final product quality. (ICH Q8, FDA PAT Guidance; ASTM E2363-06a, PQRI Robustness Paper, and EU definition)
26. Process Parameter - A process variable (e.g., temperature, compression force) that can be assigned values to be used as control levels or operating limits. (Pfizer Quality Standard definition)

27. Proven Acceptable Range - A characterized range at which a process parameter may be operated within, while producing unit operation material or final product meeting release criteria and Critical Quality Attributes. (PQRI Robustness Paper)

28. Process Robustness - Ability of a process to tolerate variability of materials and changes of the process and equipment without negative impact on quality. (ICH Q8)

29. Quality - The suitability of either a drug substance or drug product for its intended use. This term includes such attributes as the identity, strength, and purity (ICH Q8)

30. Quality Attribute - A physical, chemical, or microbiological property or characteristic that directly or indirectly relates to pre-defined product quality (safety, identity, strength, purity, and marketability of the product). (GPB Critical Process Parameters: Glossary of Associated Terms, DRAFT 05Jan'05, GAA)

31. Quality by Design - A systematic approach to development that begins with predefined objectives and emphasizes product and process understanding based on sound science and quality risk management. (ICH Q8R)

32. Quality Risk Management - A systematic process for the assessment, control, communication and review of risks to the quality of the drug (medicinal) product across the product lifecycle. (ICH Q9)

33. Quality System - The sum of all aspects of a system that implements quality policy and ensures that quality objectives are met. (ICH Q9)

34. Real Time Release - Real time release is an outcome of a control strategy in which product quality is assured for batch release through a combination of process information and input or in-process material attribute measurements during manufacturing in lieu of traditional off-line, end-product testing. (ICH Q8R)

35. Redefinition of Design Space - A redefinition of design space occurs when a manufacturing process fundamentally changes. For example, a change from wet granulation to dry granulation would require a redefinition of design space since the original process, on which the design space was based, no longer exists. A redefinition of the design space does not include an increase of knowledge around an existing process. For example, a reclassification of a parameter from CPP to $\mathrm{KPP}$ as a result of increased process knowledge is not a redefinition of design space since the subject process has not changed.

36. Requirements - The explicit or implicit needs or expectations of the patients or their surrogates (e.g., health care professionals, regulators and legislators). In this document, 
"requirements" refers not only to statutory, legislative, or regulatory requirements, but also to such needs and expectations. (ICH Q9)

37. Risk - The combination of the probability of occurrence of harm and the severity of that harm. (ICH Q9)

38. Risk Acceptance - The decision to accept risk. (ICH Q9)

39. Risk Analysis - The estimation of the risk associated with the identified hazards. (ICH Q9)

40. Risk Assessment - A systematic process of organizing information to support a risk decision to be made within a risk management process. It consists of the identification of hazards and the analysis and evaluation of risks associated with exposure to those hazards. (ICH Q9)

41. Risk Communication - The sharing of information about risk and risk management between the decision maker and other stakeholders. (ICH Q9)

42. Risk Control - Actions implementing risk management decisions. (ICH Q9)

43. Risk Evaluation - The comparison of the estimated risk to given risk criteria using a quantitative or qualitative scale to determine the significance of the risk. (ICH Q9)

44. Risk Identification - The systematic use of information to identify potential sources of harm (hazards) referring to the risk question or problem description. (ICH Q9)

45. Risk Management - The systematic application of quality management policies, procedures, and practices to the tasks of assessing, controlling, communicating and reviewing risk. (ICH Q9)

46. Risk Reduction - Actions taken to lessen the probability of occurrence of harm and the severity of that harm. (ICH Q9) 47. Risk Review - Review or monitoring of output/results of the risk management process considering (if appropriate) new knowledge and experience about the risk. (ICH Q9) 48. Robustness - The ability of a product/process to demonstrate acceptable quality and performance while tolerating variability in inputs. (PQRI Robustness Paper)

49. Stakeholder - Any individual, group or organization that can affect, be affected by, or perceive itself to be affected by a risk. Decision makers might also be stakeholders. For the purposes of this guideline, the primary stakeholders are the patient, healthcare professional, regulatory authority, and industry. (ICH Q9)

50. Trend - Tabulation or graphical plot of actual data used to determine a tendency or drift in conditions or performance.

\section{References}

1. Product Quality Lifecycle Implementation: Practical Approach to $\mathrm{QbD}$, ISPE Washington Conference, Arlington, VA, June 6-7, 2007.

2. Product Quality Lifecycle Implementation (PQLI): Practical Approach to Quality by Design, ISPE 2007 Berlin Conference, Berlin, Germany, 19-September-2007.

3. ISPE Conference on Innovation, Copenhagen, Denmark, April 711,2008

4. International Conference on Harmonisation of Technical Requirements for Registration of Pharmaceuticals for Human Use, ICH Draft: Step 1, Q8 (R1) Pharmaceutical Development Revision 1, Step 3, November 2007.

5. International Conference on Harmonisation of Technical Requirements for Registration of Pharmaceuticals for Human Use, Specifications: Test Procedures and Acceptance Criteria for New Drug Substances and New Drug Products: Chemical Substances Q6A, Step 4, 6-October-1999.

6. International Conference on Harmonisation of Technical Requirements for Registration of Pharmaceuticals for Human Use, ICH Harmonised Tripartite Guideline, Quality Risk Management Q9, Step 4, 9-November-2005.

7. International Conference on Harmonisation of Technical Requirements for Registration of Pharmaceuticals for Human Use, ICH Harmonised Tripartite Guideline, Pharmaceutical Quality System Q10, Step 2, 9-May-2007.

8. International Conference on Harmonisation of Technical Requirements for Registration of Pharmaceuticals for Human Use, ICH Harmonised Tripartite Guideline, Pharmaceutical Development Q8, Step 4, 10-November-2005.

If you would like to provide comments to the authors regarding this paper, email feedback@ispe.org and include "PQLI Criticality" in the subject line. 